\title{
Aproximación a los observatorios de medios de comunicación en colombia*
}

Approach to the media observatories in colombia

Federico Pérez Bonfante ** Ovet Uribe Metrio

Rec: $28 / 11 / 2019$ Acep: $27 / 03 / 2020$

\section{Resumen}

Este artículo da cuenta de una investigación realizada en 2019 que tuvo por objeto indagar observatorios de medios de comunicación (OMC) activos en Colombia para identificar coincidencias y diferencias en relación con cuatro asuntos puntuales: contexto en el que surgieron, base teórica, temas prioritarios de indagación y literatura publicada. Lo anterior de cara a ganar pistas para pensar un OMC para Unicatólica. Teóricamente, el artículo transita sobre las definiciones necesarias del estado del arte, observatorio de medios, relación entre medios, poderes y ciudadanía. El estado del arte se discute como una categoría central del análisis crítico. Así mismo, se asume el concepto observatorio de medios como un organismo que examina y analiza la realidad social, condicionada por los poderes políticos y económicos. En términos metodológicos, considera por base el paradigma constructivista y emplea un estudio cualitativo mediante la revisión documental como técnica de investigación. Finalmente, se obtuvieron resultados en relación con las tres primeras búsquedas señaladas, no se contó con información suficiente sobre la última que posibilitara su análisis.

Palabras clave: observatorios de medios de comunicación, democracia, poder, ciudadanía, sociedad, estado del arte.

\begin{abstract}
This article provides a detailed account of a research that took place in 2019 . The main goal of such research was to investigate active media observatories in Colombia in order to recognize differences and coincidences in four main subjects: context in which they were created, theoretical framework, subjects of investigation
\end{abstract}

* Articulo producto de investigación

** Magíster en Lingüística y Español de la Universidad del Valle, Cali Colombia. Docente del Programa de Comunicación Social y Periodismo de la Universidad Católica Lumen Gentium y miembro del Grupo de Investigación Lumen Humanitas. Correo electrónico: fpbonfante@unicatolica.edu.co; Orcid:

*** Magíster en Comunicación, Cultura y Discursos Mediáticos de la Universidad Nacional de Buenos Aires, Argentina. Docente del Programa de Comunicación Social y Periodismo de la Universidad Católica Lumen Gentium y miembro del Grupo de Investigación Lumen Humanitas. Correo electrónico: ouribe@unicatolica.edu.co; Orcid: 
and publications. This information aims at supporting the creation of a media observatory in Unicatólica. Regarding the theoretical framework of this research, this paper takes into account the necessary definitions of state of the art, media observatory, relationship between media, power and society. The state of the art is taken as a main category of critic analysis. Further, this article understands the concept of media observatory as an organism that investigates and analyzes social reality, always conditioned by politic and economic powers. In terms of the methodology and taking into account the constructivist paradigm, this is a research based on a qualitative study that employs documental review as an investigation technique. Lastly, results were obtained regarding the first three research subjects but not for the last one, due to the fact that no information was found.

Keywords: media observatory, democracy, power, citizenship, society, state of the art.

\section{Introducción}

En el mundo contemporáneo, la crisis de la relación entre medios de comunicación, ciudadanía, democracia y poder se ha agudizado. Resulta cada vez más evidente la estrecha relación de complacencias entre los medios de comunicación y los poderes dominantes (económico y político) con la consecuencia lógica de un detrimento para la ciudadanía y la democracia. En palabras de Herrera (2016, p. 46):

[... La sumisión de la industria periodística a determinados intereses ideológicos ha sido también motivo de más de una sospecha: pese a que los medios gusten de verse a símismos como vigiladores [sic] de las malversaciones del poder, en la mayor parte de las ocasiones, la misma configuración del sistema mediático origina una dependencia recíproca entre poder y medios en la que, nuevamente, el interés del público parece quedar relegado.
En razón a esta crisis, de acuerdo con Castellanos (2010), desde los años cuarenta del siglo pasado inicia la historia de los observatorios, historia que no se ha detenido hasta hoy en día, pues como ya se indicó, la crisis se ha agudizado con el pasar del tiempo. Un momento icónico más próximo a tiempos actuales fue el Foro Social Mundial de Porto Alegre en 2002 en el que el destacado intelectual y director de Le monde diplomatique, Ignacio Ramonet, llamó a

[... consolidar en el orbe un quinto poder a través de la constitución de un Observatorio Internacional de Medios de Comunicación, es reconocida como un importante incentivo a partir del cual se iniciaron muchos de estos proyectos. (Propuesta que se materializa al año siguiente en el mismo evento]. (Castellanos, 2010).

La importancia de vigilar a los medios de comunicación, como ya ha quedado sugerido, es restablecer la relación de control de estos con respecto a los poderes económicos y políticos, de suerte tal que la ciudadanía se encuentre bien informada de cara a su participación y fortalecimiento de una sociedad democrática. Esto es particularmente importante en un país como Colombia en el que, con frecuencia, se presentan casos de censura a la prensa por parte de los gobiernos de turno (Publimetro, 2019; El Espectador, 2019) y de alta concentración del poder económico sobre los medios de comunicación (Laszorillas, 2015).

Dado lo anterior y en el ánimo de pensar un Observatorio de Medios de Comunicación para Unicatólica, el asunto fundamental de esta investigación fue reconocer características básicas de observatorios de comunicación social y periodismo existentes en Colombia. Para alcanzar este propósito se decidió, en primer lugar, identificar el contexto histórico en el que surgen los observatorios; en segundo lugar, reconocer las definiciones del concepto observatorio de medios de comunicación y periodismo que evidencien 
las perspectivas teóricas desde las cuales se han fundado. Asimismo, se indagaron los campos de estudio de interés que presentan y finalmente, se tuvo la intensión de inventariar las investigaciones y publicaciones académicas llevadas a cabo. Sin embargo, este último objetivo no se logró llevar a cabo por la escasa información obtenida de los tres observatorios objeto de estudio.

\section{Antecedentes}

Para construir un marco de comprensión histórica que permitiera la aproximación que pretendía el estudio se seleccionaron cuatro artículos que dan cuenta de estados del arte de observatorios de medios de comunicación. Estos abarcan un periodo de ocho años (2008-2016) y se originaron en España, Ecuador, México y Colombia. Uno de los criterios que se tuvo en cuenta fue la amplitud de los observatorios considerados en las investigaciones. El artículo que presenta los resultados del estudio de España tuvo como referencia, por ejemplo, 28 observatorios situados en América Latina más 28 localizados en el país ibérico. A continuación se presentan los resultados de haber cruzado estos cuatro artículos a la luz de los siguientes temas: razones por las que emergieron los observatorios; definiciones y tipos de observatorios; campos de conocimiento en los que se sitúan; y finalmente, ámbitos de acción, dificultades y retos de los OMC.

En relación con las causas que dispararon el surgimiento de los observatorios, los estudios coinciden en que los medios no cumplen con su lugar en el marco de una democracia liberal que consiste en vigilar a los poderes (políticos y económicos), representar a la ciudadanía y mantenerla informada. Así mismo, coinciden en que hay poderes económicos y agendas políticas que se han hecho a los medios de comunicación. El artículo que trata sobre el caso mexicano, por ejemplo, lo señala de la siguiente forma: los Observatorios de Medios en México surgen en un contexto caracterizado por la alta concentración mediática, la colusión entre los empresarios de los medios y el Estado, así como por las constantes críticas en torno a la responsabilidad social de los medios [...] Son también el resultado de una mayor concientización por parte de las elites políticas, sectores especializados y ciudadanos en general acerca del creciente poder de los medios en las democracias contemporáneas, el cual no siempre se acompaña de un ejercicio responsable de sus funciones. (Sánchez y Díaz, 2014)

Y agrega inmediatamente:

Aunque los medios son usualmente considerados como un actor fundamental en la supervisión y el control de las acciones de los gobiernos en la teoría liberal democrática (la hipótesis del perro guardián [watchdog] y en la literatura sobre rendición de cuentas societal [...], en las últimas décadas se han incrementado las críticas provenientes de académicos y diversos actores de la sociedad civil hacia su comportamiento. (Sánchez y Díaz, 2014, p. 208)

Ahora bien, los estados del arte consultados también intentaron hallar una definición de observatorios. No obstante, no se pudo obtener una respuesta, es decir que no existe una concepción única de observatorios de medios; la diversidad de orígenes de estos y de los diferentes tipos de agentes que los gestionan (administraciones públicas, universidades, sociedad civil organizada, colectivos de periodistas, etc.) desencadenan una heterogeneidad conceptual. Esta diversidad alrededor del concepto de los observatorios se expresa de manera clara en un apartado del artículo de Castellanos (2010), quien apela a un conjunto de autores que han trabajado este asunto y que podría quedar listado de la siguiente manera: 
Los observatorios de medios han sido nombrados como: experiencia; metodología; institución; proyecto; instancias de supervisión mediática; "una estrategia de observación y análisis para hacer seguimiento a los medios masivos"; "experiencias de participación social, de ejercicio de los derechos a la comunicación, la información y la libertad de expresión"; iniciativas y proyectos que van desde observatorios de medios y blogs hasta sites personales en la web; " metáforas recientes de procesos sociales y comunicativos fuertemente relacionados con movimientos de democratización de la sociedad"; espacios de supervisión del desempeño mediático, en especial del campo noticioso; laboratorios de experimentación para analizar medios masivos de comunicación y sus efectos en la sociedad. Esta ausencia de consenso, afirma la autora, "no coloca límites ni epistemológicos ni prácticos, incluso diversifica la experiencia a partir de las posibilidades que ofrece Internet." (Castellanos, 2010, p. 11).

En relación con los tipos de observatorios, resulta obvia la conclusión a la luz del primer asunto abordado (los contextos en los cuales surgen los OMC). Tres de los cuatro artículos, aquellos que dan cuenta de las experiencias en América Latina, coinciden en que los observatorios son fiscalizadores; es decir, su función fundamental es vigilar a los medios. No obstante y aunque el estado del arte que puso la lupa en las experiencias españolas también lo reconoce así, existen también observatorios estadísticos que se alejan diametralmente de los anteriores, a saber: "se dedican más bien a la recolección, categorización y difusión de datos beneficiosos para el mercado y que suelen estar a cargo de Administraciones públicas" (Rosique y Barranquero; 2016, p. 447).

Excepto los recién nombrados observatorios estadísticos, los demás (fiscalizadores) se enmarcan en el campo de conocimientos de las ciencias sociales y la literatura consultada coincide y es enfática en la crisis de la relación medios de comunicación y democracia. Rosique y Barranquero (2016) señalan que existen dos campos de estudio: 1. Medios y democracia y 2. Observatorio de medios. En relación con el primero, destaca la referencia al libro de Pierre Rosanvallón publicado en 2007, La contrademocracia. La política en la era de la desconfianza. De este autor retoman la característica de fiscalización como uno de los elementos que define a las democracias contemporáneas y que supera la concepción restringida que la define solo como pueblo que vota.

Igualmente, Rosique y Barranquero (2016) señalan que en el contexto de desafección política y pérdida de legitimidad de muchas instituciones "los medios deben de jugar un rol de observación crítica, desconfianza y de 'tercero investigador' con respecto al resto de organizaciones y a fin de implicarlas en la defensa del bien común". ( $\rho .445)$ Por otro lado, los autores recurren a G. Weill para vincular la definición de medios de comunicación consignada en el libro El periódico: origenes, evolución y función de la prensa periódica publicado en 2007, a saber: "han sido definidos [los medios de comunicación] como el 'cuarto poder' por cuanto su misión consiste en fiscalizar al poder económico y político y representar a una ciudadanía de la que, en último término, son voceros y portavoces" (p. 444).

Herrera (2016) advierte una objeción que también se encuentra más adelante en Rosique y Barranquero:

Sin embargo, a pesar de la importante labor que llevan a cabo los medios, existe también hoy una percepción generalizada de que [e]stos no están haciendo las cosas como deberían. Las objeciones planteadas a un uso poco responsable de los medios se han concretado en numerosos ataques tanto al mismo proceso noticioso como al resultado final a que este proceso da lugar. (2008, p. 45) 
Ahora bien, en relación con lo que este documento plantea que es más relevante para efecto de apoyo a esta investigación, los artículos consultados arrojaron elementos significativos en relación con los ámbitos de acción del trabajo de los OMC. Todos los observatorios coincidieron en que se lleva a cabo un trabajo de investigación a los medios objeto de análisis que se traduce en informes. Si el OMC está anclado al escenario universitario, los informes son académicos. Uno de los problemas revelados es que los observatorios vinculados al mundo universitario se centran en medios de carácter nacional y descuidan así los contenidos regionales y locales. Así lo deja ver Castellanos: "las universidades del centro hacen una mirada general sobre el país, pero se enfocan poco en las regiones; las regiones, en cambio, sin dejar de pensar lo local, están al corriente de lo que acontece desde el centro." (2010, p. 14).

Finalmente, en relación con las dificultades y retos, se podría sintetizar a través del siguiente listado ofrecido por Rosique y Barranquero (2016, pp. 462-463) y que recoge muy claramente el panorama ofrecido en los cuatro artículos considerados:

1. [los observatorios] se caracterizan por una actividad investigadora irregular, inestable $y$, en algunas ocasiones, anecdótica.

2. Son escasos los que están cumpliendo con [...] las labores de pedagogía, intermediación con la ciudadanía, y movilización ciudadana para la mejora de las políticas públicas.

3. La sociedad civil no se tiene en cuenta en la vida diaria del observatorio, sino que es más bien 'representada'.

4. La conciencia de grupo y de red es nula entre los observatorios españoles. [...] La sostenibilidad de los observatorios pasa, no solo por un remanente constante de fondos y personal especializado, sino también por la creación de redes de confianza y cooperación que permitan comparar y poner en común resultados, organizar acciones conjuntas para la divulgación de resultados, y armar agendas políticas transformadoras.

5. Su impacto es limitado en la esfera pública y en la propia representación en los organismos públicos encargados de la formulación de políticas comunicacionales.

\section{Consideraciones conceptuales}

Esta investigación transita sobre cuatro elementos que fundan su postura teórica y determinan sus intereses:

- Investigación del estado del arte

- Conceptualización de observatorio de medios de comunicación y periodismo

- Relación entre medios de comunicación y poder

- Medios de comunicación, poder político y económico y ciudadanía

Al decir de Guevara (2016), el estado del arte es una categoría central y deductiva que se aborda y se propone como estrategia metodológica para el análisis crítico. Dicho análisis recurre a postulados metodológicos de la hermenéutica y la heurística. Los postulados de la primera suponen la capacidad de explicar y traducir las relaciones entre el hecho y el contexto; los de la segunda comprenden la capacidad de realizar la búsqueda de información necesaria para los procesos investigativos (Londoño, Maldonado y Calderón 2014.)

Este tipo de investigaciones del estado del arte reconoce tres aproximaciones conceptuales que 
conducen a la sistematización y construcción del sentido. Es una investigación documental, lo que revela que su estudio es metódico y responde a objetivos definidos. Cifuentes, Osorio y Morales (citados por Guevara 2016) piensan que, a su vez, "sirve de base para la comprensión del problema, la definición de nuevos hechos o situaciones problemáticas, la elaboración de una hipótesis o la orientación a nuevas fuentes de investigación en la construcción de conocimiento."

Es también una revisión de propuestas en tanto insiste, según Uribe (2005), en una mirada sobre la producción previa. Fruto de esta estrategia de mirada múltiple, Calvo (citado por Guevara 2016) recuerda que se construyen nuevos campos teóricos y metodológicos nacidos de la revisión crítica del pensamiento previo.

Finalmente, Guevara (2016) reconoce que existen dos posturas dentro de las investigaciones sobre el estado del arte: una positivista que entiende la realidad por fuera del individuo, que se puede verificar y describir a partir de estadísticas y otra postura constructivista que entiende que la realidad es una construcción colectiva y que por ende es múltiple y variable. En esta postura se valoran las voces diversas dentro de la investigación y durante la revisión documental. Es bajo esta concepción epistemológica que se enmarca esta investigación, una investigación que valora el marco histórico y social de los textos.

De otro lado, el concepto de observatorio, como se aclaró anteriormente, es confuso. La construcción teórica es limitada y carece de acuerdos. Se cuenta con documentos institucionales que los fundan desde un interés particular sin establecer lenguajes comunes sobre el concepto. La Universidad Sergio Arboleda, por ejemplo, entiende su Observatorio como un "espacio académico que busca ejercer como veedor de las relaciones de poder que se generan entre el gobierno, los medios, los líderes de opinión, las fuentes y los ciudadanos." Queda establecido el carácter vigilante frente a las relaciones de poder que se tejen con los medios como bisagra.

El Observatorio de Medios, Político, Social y Cultural de la Unión de Trabajadores de prensa de Buenos Aires (UTPBA) entiende que la rigurosidad de su práctica nace de la interpretación de "los modos en que pujan las voces que luchan contra tanta injusticia en el marco de las relaciones de dominación económica, política, social y cultural existentes." El espacio de la UTPBA propone la resignificación de los relatos/técnicas periodísticas (manipulación, objetividad, etc.).

Para la Universidad de Pamplona, en Norte de Santander, su observatorio es un espacio comunitario de abordaje de la realidad mediatizada y tiene como propósito fundamental "la mirada de los contenidos en el contexto de las teorías de la comunicación, el papel de la comunicación como motor de los procesos de educación y la construcción de participación ciudadana" (Observatorio de medios, s.f.).

En el terreno de la relación entre poder y medios de comunicación Becerra y Mastrini (2006, p. 2) entienden que

El abordaje del estudio de la concentración de la propiedad de los medios de comunicación debe considerar, entendemos, tanto su dimensión económica como política. En el plano económico, la producción cultural comparte algunas características con los bienes de consumo. Su cualidad esencial, de la que deriva su valor de uso, es ser un bien inmaterial, un contenido simbólico transportado por algún soporte.

Al ser la información un bien inmaterial que no desaparece cuando se consume, exige ser renovado incesantemente y a bajo costo, lo 
que permite la expansión y concentración de la industria. Este crecimiento exponencial lo vincula a un necesario análisis sociopolítico y no solo económico.

Desde un punto de vista sociopolítico, un elemento central está constituido por el pluralismo informativo y cultural. Una de las claves de la participación ciudadana en la definición de las principales tendencias de la sociedad es tener la posibilidad de acceder a una variada gama de productos culturales y opiniones. La concentración de la propiedad de los medios limitaría esta variedad y existen diversos ejemplos de intervención de los Estados Nacionales con el objetivo de fomentar la pluralidad. (Becerra y Mastrini, 2006, p. 3)

Esta alta rotación del bien inmaterial, junto a un modelo económico funcional al sistema, permitió la privatización, concentración y, con referencia a Mcluhan (1964, p. 20), también permitió y agenció una clara influencia en la construcción de imaginarios sociales.

En una cultura como la nuestra, con una larga tradición de fraccionar y dividir para controlar, puede ser un choque que le recuerden a uno que, operativa y prácticamente, el medio es el mensaje. Esto significa simplemente que las consecuencias individuales y sociales de cualquier medio, es decir, de cualquiera de nuestras extensiones, resultan la nueva escala que introduce en nuestros asuntos cualquier extensión o tecnología nueva.

En esta misma línea De Moraes, Ramonet y Serrano (2013) identifican, a su vez, que los medios tienen la capacidad de fijar de fijar sentidos e ideologías, formar opiniones y trazar líneas predominantes del imaginario social. Hay una procura por la mercantilización del bien inmaterial que usa como instrumento al medio.
[...] el sistema mediático infunde y celebra la vida para el mercado, la supremacía de las seducciones consumistas, el individualismo y la competencia; la existencia subordinada al mantra de la rentabilidad. La glorificación del mercado consiste en presentarlo como el medio más adecuado de traducir anhelos de la sociedad, como si solamente él pudiera convertirse en instancia de organización societaria. Un discurso que no hace más que realzar y profundizar la visión, claramente autoritaria, de que el mercado es la única esfera capaz de regular, por símisma, la vida contemporánea. (2013, p. 13)

Para terminar, bajo esta premisa que vincula medios, poder y ciudadanía cabe insistir con Ramonet (De Moraes et al., 2013) en una primera paradoja mediática

Los medios dominantes pertenecen a grupos importantes en el sector del mercado, ¿puede creerse que serán muy críticos con la globalización, con el neoliberalismo, cuando son actores centrales de esas dos dinámicas? Evidentemente hay poca posibilidad de que lo sean. Por consiguiente, en realidad hoy en día los grupos mediáticos se están comportando como el brazo ideológico de la globalización. Para decirlo de otra manera, utilizando el concepto althusseriano, son la herramienta ideológica de la globalización.

\section{Metodología}

Dado que la investigación base de este artículo tuvo como fundamento el paradigma constructivista, la perspectiva cualitativa resultó más pertinente, pues los estudios que hacen uso de este enfoque abarcan "casos particulares y procesos singulares que proporcionan elementos significativos para la comprensión de la realidad" (Melero, 2011, p. 343). Es de anotar, además, que el corpus objeto de estudio estuvo constituido por 
documentos escritos y que "El estudio cualitativo busca la comprensión de los fenómenos en su ambiente usual, desarrollando la información basada en la descripción de situaciones, lugares, periódicos, textos, individuos, etc." (Ramos, 2015, p. 15, énfasis añadido). Finalmente, se asumió la perspectiva cualitativa para la investigación dado que en esta "El investigador emplea técnicas como la observación no estructurada, entrevistas abiertas, revisión de documentos, discusión de grupo, evaluación de experiencias personales o registro de historias de vida" (Ramos, 2015, p. 16, énfasis añadido).

Debido a que el fenómeno de indagación fue de carácter social, abordado cualitativamente sin la pretensión -como en el positivismo- de establecer leyes o generalizaciones, este proyecto de investigación se inscribió en el paradigma constructivista de investigación, como ya se había anunciado. La idea anterior la reafirma Ramos (2015, p. 14) al retomar a Hernández en el texto Metodología de la investigación (2010) cuando sostiene que una de las principales afirmaciones de este paradigma es que "Los resultados no pueden ser generalizados en forma ajena al contexto y el tiempo". Asimismo, dado que el fenómeno objeto de estudio estuvo mediado y construido en el uso del lenguaje, este paradigma resultó preciso, toda vez que, según Ramos (2015), al eludir a Berger y Luckman, señala que:

[...] la posición teórica que adopta el paradigma constructivista social se basa en el principio de que el saber del mundo real se construye en base a procesos de interaccionismo social y de movilización de recursos persuasivos y representacionales. (p. 14)

Poco después cita el artículo de Gergen (2007) "Construccionismo social. Aportes para el debate y la práctica" y añade que: [...en] el constructivismo se brinda gran interés al cómo se producen los significados y su utilización mediada por las convenciones lingüísticas dentro de los diversos contextos sociales o culturales, donde los individuos otorgan significados dentro de marcos de referencia interpretativos, basados en la historicidad y en lo cultural. (p. 14)

El corpus objeto de estudio estuvo constituido por tres documentos a los que se les aplicó el mismo instrumento. Cada uno de ellos estuvo vinculado a uno de los observatorios de medios de comunicación (OMC) indagados. Así las cosas, para el OMC del Caribe colombiano se tuvo en cuenta el documento académico-institucional que fue el fundamento para su creación (Coronel, Herrera y Del Castillo, 2016). Para el caso del OMC de la Universidad de la Sabana, el instrumento se aplicó a la información publicada en la página web de esta institución (Universidad de la sabana, s.f.). Finalmente, el OMC de la Universidad Santiago de Cali fue el único del cual se obtuvo respuesta directa sobre el instrumento. El análisis se desarrolló con el cruce de información de los tres OMC a partir de lo arrojado por el instrumento, se hallaron coincidencias y diferencias.

\section{Resultados}

En relación con el objetivo específico n.ำ 1 , se hallaron los siguientes resultados. Se inicia con el Observatorio de Medios del Caribe colombiano, agenciado por la Universidad Sergio Arboleda, sede Santa Marta:

Estas iniciativas también se han visto motivadas por la percepción de que en la actualidad los medios masivos han dejado atrás su compromiso de entregar información veraz e imparcial, y de ser el Cuarto Poder, en cuanto fiscalizadores de los demás poderes (ejecutivo, legislativo y judicial). Por el contrario, debido a diferentes circunstancias, se ha confundido con 
ellos y en ocasiones ha perdido su norte y la responsabilidad en la emisión y producción de contenidos. En efecto, estos ya no son pensados tanto para responder a las necesidades de información, formación y entretenimiento de los receptores, sino más para satisfacer y complacer a los poderes establecidos. (Coronel et al., 2016, p. 16)

\section{Y más adelante agrega:}

Problemáticas como la concentración de la propiedad de los medios, los estrechos vínculos entre ellos y los poderes políticos y económicos son solo dos grandes situaciones que aún caracterizan las dinámicas de la comunicación en la región norte del país, y por ello todavía se requiere una reflexión constante que posibilite discutir y debatir la responsabilidad que la prensa, la radio y la televisión, producidas en esta zona del país tienen con sus públicos y audiencias. De ahí el aporte que puede realizar el grupo de investigación Comunicación y Sociedad, a través de un Observatorio de Medios del Caribe colombiano." (Coronel et al., 2016, p. 18)

En el caso del Observatorio de la Universidad de La Sabana, cuya información se obtuvo a través de la página web de esta institución, el contexto de surgimiento tiene que ver con tres acontecimientos, pero nuevamente ligado al incumplimiento del rol de los medios de comunicación, a saber:

Fueron tres los momentos que promovieron la preocupación de los investigadores del observatorio por el poco ético cubrimiento de las noticias sobre los acontecimientos que vivía el país: el comienzo de la televisión privada en el país, con una gran inversión que permitió llevar la señal de sus transmisiones a casi todo el territorio colombiano y entregar la información en tiempo real desde el lugar de los acontecimientos; el cubrimiento del terremoto de Armenia, Quindío, en el que murieron mil personas y otras cinco mil resultaron heridas; y el episodio de la famosa 'silla vacía', cuando el presidente Andrés Pastrana pensó que iniciaría negociaciones con los grupos subversivos para lograr la paz y los medios de comunicación dudaban entre registrar la información o ser parte activa como garantes del proceso: ¿cuál era su papel? Finalizaba el milenio [...] En ese momento los docentes-investigadores de la Facultad de Comunicación Social dijeron 'No más'; investigaron y movilizaron a los medios logrando su compromiso para hacer un alto en el camino y reflexionar. (Universidad de La Sabana, s.f.)

En el caso del Observatorio de la Universidad Santiago de Cali, que entregó la información a través del diligenciamiento del instrumento, se puede conectar con las respuestas dadas a través de una pista en sus respuestas: autocensura. Obsérvese:

En 2002 publicó un análisis de la Prensa Colombiana con el docente German Ayala sobre Plan Colombia; fue un seguimiento de 6 medios durante un año con un libro publicado Plan Colombia un año de Autocensura. Como resultado se creó el Observatorio de Medios en la USC.

En relación con el objetivo específico n. 2 , no se obtuvo información del Observatorio de la Universidad de La Sabana, pero sí de aquel de la Universidad Santiago de Cali (Usaca) y del Observatorio de medios del Caribe colombiano. En ambos casos la información da cuenta del campo de las ciencias sociales como encuadre teórico y conceptual general de orientación. En lo particular, en medio de este campo, se señalan teorías ligadas al campo del lenguaje desde una perspectiva social. En el caso del observatorio de la Usaca, se consideran: 
Los estudios norteamericanos sobre estudios de los mass media surgidos de la escuela de Chicago y Opinión Pública (Wolf) con bases en estudios empíricos y semióticos bajo la influencia del pensamiento de la escuela de Tartú (Lotman). Por otra parte, los análisis del discurso de corte sociolingüístico con Van Dijk, Habermas y la escuela de Praga.

Por su parte, el Observatorio de Medios del Caribe estima que:

La práctica periodística de hoy sobrepasa las funciones originarias del periodismo liberal. Así, ya no se puede observar la formulación de la noticia como algo ajeno a la construcción de la realidad social, [...] Es de esta forma que los escenarios de estudio dentro de las ciencias sociales [...] comienzan a recaer en elementos claves que son parte de los procesos de convivencia y que se manifiestan en las luchas, negociaciones, tensiones y hegemonías que se viven dentro de los procesos [de] comunicación de los órdenes informativos contemporáneos. (Coronel et al., 2016, p. 95)

En relación con el objetivo n. 3 el abanico de temas de interés del observatorio de la Universidad de La Sabana (s.f.) quedan revelados a través de sus líneas de investigación:

1. Comunicación pública y participación ciudadana

2. Libertad de prensa y derecho a la información

3. Tratamiento periodístico de la información

4. Cultura de masas e industrias culturales

5. Formatos de entretenimiento de medios

6. Consumo de medios

\section{Comunicación política}

8. Educación de audiencias

9. Comunicación para el desarrollo

10. Calidad de la información periodística

El Observatorio de Medios del Caribe colombiano parce tener una tendencia más crítica social y política en relación con la estructura de poder regional, pues afirman que:

No obstante la complejidad y pluralidad de los medios de comunicación de la región, es posible que la Escuela de Comunicación Social y Periodismo de la Universidad Sergio Arboleda, sede Santa Marta, impulse una iniciativa de este tipo, pues aún son muchos los temas sensibles sobre medios y sociedad que se pueden abordar a partir de una vigilancia juiciosa y sistemática. Particularmente los relacionados con la marginalidad, la pobreza y la exclusión, que son las temáticas que desde hace siete años el grupo de investigación Comunicación y Sociedad ha venido trabajando [...) (Coronel et al., s.f., énfasis añadido)

Y agregan pocas líneas después:

Problemáticas como la concentración de la propiedad de los medios, los estrechos vínculos entre ellos y los poderes políticos y económicos son solo dos grandes situaciones que aún caracterizan las dinámicas de la comunicación en la región norte del país, y por ello todavía se requiere una reflexión constante que posibilite discutir y debatir la responsabilidad que la prensa, la radio y la televisión, producidas en esta zona del país tienen con sus públicos y audiencias. De ahí el aporte que puede realizar el grupo de investigación Comunicación y Sociedad, a través de un Observatorio de 
Medios del Caribe colombiano." ((Coronel et al., s.f., p. 18, énfasis añadido)

Finalmente, el Observatorio de Medios de la Universidad Santiago de Cali señala que sus objetos de estudio han versado sobre:

1. Análisis de rutinas periodísticas

2. Calidad informativa

3. Agenda Setting y framing

4. Análisis de los discursos

5. Opinión pública

6. Temas étnicos y de género

7. Análisis de medios digitales y nuevos discursos

8. Tendencias y nuevas dinámicas discursivas de la comunicación

Ahora bien, en relación con la producción de conocimiento, solo se obtuvo información de uno de los tres observatorios indagados, el de la Universidad del La Sabana. La información obtenida a partir de su página web es la siguiente:

\section{Capítulos de libros}

- "El plebsicito del 2 de octubre en Colombia: El divorcio entre la opinión publica y la opinión publicada"

- "Movimientos en (des-)acuerdo con la red ¿mercantilizando o haciendo común internet?"

- "Rethinking convergence: A new word to describe an old idea"
"El plebiscito del 2 de octubre a través de las pantallas: Lo que va del centro a la 'periferia'. Análisis del cubrimiento de noticias RCN, Caracol noticias, City noticias y Capital noticias"

\section{Artículos en revistas indexadas}

- El slacktivismo como recurso de movilización en redes sociales: El caso de \#BringBackOurGirls"

- "Hacia la comprensión de la naturaleza transnacional de las audiencias televisivas: Imaginarios, desterritorialización, hibridación, globalización"

- "Factores que facilitan la generación de 'engagement' con programas de televisión: exploración cualitativa desde la identidad del canal, las características del producto y la valoración de las audiencias"

- "El efecto dominó de las revueltas sociales de 2011 visto desde la prensa de referencia colombiana"

La fotografía y el texto en la construcción del framing de la Primavera Árabe: un análisis del cubrimiento y tratamiento informativo de la prensa de referencia colombiana, en 2011"

\section{Discusión de los resultados}

En el caso de los resultados de cara al objetivo específico $n . \underline{1}$, estos confirman lo señalado por Herrera (2008) en el sentido de reconocer que los medios de comunicación no cumplen cabalmente con el lugar funcional que les corresponde en el marco de sociedades democráticas liberales. En palabras de Díaz Nosty, citado por Rosique y Barranquero, efectivamente existe una crisis del "cuarto 
poder" que ha "provocado una creciente desafección ciudadana hacia el sistema de medios" (2016, p. 445). En nuestro contexto, el progresivo distanciamiento de la ciudadanía frente a los medios ha quedado en evidencia a través de un estudio del Observatorio de la Democracia (2017) que señala que:

En el caso colombiano, los medios de comunicación hasta el 2012 habían sido una de las instituciones públicas con más prestigio histórico, según datos del Barómetro de las Américas-LAPOP, realizado por el Observatorio de la Democracia de la Universidad de los Andes. Sin embargo, en el 2013 la confianza de los ciudadanos hacia los medios de comunicación sufrió un desplome. Para el 2012, el 60,5\% de los colombianos manifestó confiar en los medios de comunicación, mientras que entre 2013 y 2016 este porcentaje se redujo a casi la mitad, llegando a 35,8\%.

\section{Y agrega:}

En el 2016 la baja confianza hacia esta institución fue generalizada en el territorio nacional. En concreto, en las principales regiones del país, menos de la mitad de los habitantes manifestó confiar en los medios de comunicación [...]. Esta situación es preocupante debido a que históricamente fueron una de las instituciones con más prestigio a nivel nacional y al papel clave que tienen en una democracia.

En relación con los resultados hallados a la luz del objetivo específico n. 2 se debe comprender que las ciencias sociales son el campo epistemológico en el cual tienen lugar tanto los medios de comunicación como los observatorios. Esto supone recabar un conjunto de teorías sociales, políticas, del lenguaje y la comunicación que den cuenta de las relaciones entre estas dimensiones humanas que dan lugar a la construcción de las sociedades. Por ello, un segmento de la respuesta dada por el Observatorio de la Universidad Santiago de Cali apunta hacia "van Dijk, Habermas y la escuela de Praga". En nuestro contexto de investigaciones académicas, la apelación a los estudios de Teun A. van Dijk, desde la perspectiva del análisis crítico del discurso en particular, han constituido una tradición y escuela de estudio, no solo en Colombia sino en América Latina y Europa.

Ahora bien, en relación con los temas de interés de los observatorios indagados (objetivo específico n.․ 3), el abanico es amplio, da cuenta de las coordenadas de contexto señaladas en el primer resultado y, también, hay matices que los diferencian. Es posible que esto último se explique en razón a los antecedentes diversos de cada uno de los observatorios. Por ejemplo, en el caso del Observatorio de la Universidad de La Sabana (s.f.), señalan en su historia de vida que:

\begin{abstract}
Asícomo los periodistas en ejercicio deben hacer seguimiento a las historias que publican, los investigadores del Observatorio se convirtieron de nuevo en los más asiduos consumidores de noticias, para comprobar si se estaban cumpliendo los acuerdos, lo que los ha llevado más allá de lo que leen, escuchan y ven por la tele. Así surge el análisis sobre la libertad de prensa, que se convertiría en una de las cuatro líneas de investigación del grupo.
\end{abstract}

Otro elemento que puede entrar a colación en la amplitud de los temas lo revela el Observatorio de Medios del Caribe colombiano, y tiene que ver precisamente con la geografía política de las regiones. Vale la pena mencionar los que este Observatorio afirma:

Problemáticas como la concentración de la propiedad de los medios, los estrechos vínculos entre ellos y los poderes políticos y económicos 
son solo dos grandes situaciones que aún caracterizan las dinámicas de la comunicación en la región norte del país [...] (Coronel et al., 2016).

Así como los antecedentes constituyen elementos que matizan diferencias, también hay lugares comunes, por ejemplo, las líneas de investigación n. 01 y 2 del Observatorio de la Universidad de La Sabana coinciden con el tema de interés n.․ 5 del Observatorio de la Usaca.

Finalmente, no es posible construir una discusión ni recomendación en relación con los resultados ligados al objetivo n. 4 dado que no se cuenta con información suficiente que posibilite una comprensión del asunto en particular (las publicaciones). Como ya se había señalado, solo fue posible identificar la producción de conocimiento de uno de los observatorios, lo cual no permite hacer un contraste.

\section{Conclusiones}

El desarrollo de esta investigación permite concluir la pertinencia académica, social y política de los observatorios de medios de comunicación, en especial los fiscalizadores, como instancias de la sociedad civil para la vigencia de la democracia. La demostrada cercanía entre poder político, económico y los medios de comunicación en Colombia, que ha conducido a la cada vez menor credibilidad de la ciudadanía hacia esta institución (medios de comunicación) exige esfuerzos para recuperar su lugar social y político. Esta pertinencia se hace aún más urgente en razón al escaso número de observatorios a nivel nacional, especialmente a nivel regional y local, puesto que tan solo hay uno en Cali, a pesar del número de universidades que ofrecen el programa de Comunicación Social y Periodismo. Lo anterior exhorta a plantearse la pregunta sobre la razón por la cual las universidades en particular, pero también la sociedad en general no ha considerado la vigilancia de los medios de comunicación como prioridad democrática.

En términos académicos, la pertinencia se refiere a la posibilidad de alimentar los desarrollos teóricos de la relación lenguaje poder - sociedad en el marco de la perspectiva discursiva del lenguaje, la cual es base del análisis del discurso (AD) y del análisis crítico del discurso (ACD). Lo anterior a partir del estudio de casos a nivel local, regional y nacional en el marco de un observatorio de medios de comunicación (OMC) de Unicatólica. Asimismo, la existencia de OMC en términos metodológicos supone posibilidades para implementar diseños metodológicos existentes, ajustar en otras oportunidades y crear nuevos, en el caso de esta investigación, a partir de los aportes de la linguíística textual, el AD y el ACD, perspectivas pocas veces consideradas por los programas de Comunicación Social y Periodismo para la investigación.

Asimismo, la emergencia de medios de comunicación por fuera de los tradicionales dado al desarrollo de nuevas tecnologías de la información y la comunicación, entre las que se incluyen redes sociales, es un campo que abre posibilidades parainvestigar las reconfiguraciones en los modos de informarse y la reconstrucción de ciudadanías y, quizá, de la propia democracia. Aquí los observatorios también tienen un lugar en tanto escenarios de investigación. De hecho, como se constató con los antecedentes consultados, algunos observatorios tienen por objeto de estudio el periodismo ciudadano realizado a través de plataformas como Facebook y YouTube. Sin embargo, los OMC objeto de este estudio no dan cuenta de manera destacada de investigaciones relacionadas con este nuevo campo de la comunicación y ejercicio del periodismo.

Finalmente, de este estudio se concluye una urgencia adicional: la necesidad de fortalecer 
el trabajo colaborativo entre los observatorios activos que permita, no solo generar ganancia para sí en términos académicos, sino además para la incidencia en políticas de medios a nivel nacional, como es el propósito de algunos observatorios por fuera de Colombia.

\section{Referencias}

Coronel Noguera, H. J., Herrera Delgams, L., y Del Castillo Gutiérrez, J. (2016). Observatorio de medios del caribe colombiano. Universidad Sergio Arboleda. https://repository.usergioarboleda. edu.co/bitstream/handle/11232/936/ Observatorio\%20de\%20medios\%20del\%20 Caribe.pdf? sequence=1\&isAllowed=y

Castellanos Díaz, ). (2010, agosto-octubre). Los observatorios de medios: bastiones de la investigación mediática. Razón y Palabra, (73). http://wwww.razonypalabra.org.mx/N/N73/ Varia73/23Castellanos_V73.pdf

De Moraes, D., Ramonet, I., y Serrano, P. (2013). Medios, poder y contrapoder, de la concentración monopólica a la democratización de la información. Editorial Biblos.

El Espectador (2019, enero 24). Juan Pablo Bieri renuncia a RTVC por escándalo de censura a Los Puros Criollos. https://uww .elespectador.com/noticias/ nacional/juan-pablo-bieri-renuncia-rtvc-porescandalo-de-censura-los-puros-criollosarticulo-835803

Guevara, R. (2016, julio-diciembre). El estado del arte en la investigación: ¿análisis de los conocimientos acumulados o indagación por nuevos sentidos? Revista Folios, (44), 165-179. http://wwww.redalyc. org/pdf/3459/345945922011.pdf

Herrera Damas, S. (2008, enero-junio). Vigilando a los medios: observatorios y defensores de la audiencia en el contexto del media criticism. Anagramas Rumbos y Sentidos de la Comunicación, 6(12), 43-59.

Las2orillas (2015, octubre 15). ¿De quién son los medios en Colombia? Las dos orillas. https://uruw. laszorillas.co/de-quien-son-los-medios-decomunicacion-en-colombia/\#

Londoño, O., Maldonado, L., y Calderón, L. (2014). Guía para construir estados del arte. International corporation of networks of knowledge.

Mastrini, G., y Becerra, M. (2017). La concentración infocomunicacional en América Latina 20002015. Universidad Nacional de Quilmes Editorial. https://www.observacom.org/wp-content/ uploads/2019/09/La-concentracio\%cc\%81ninfocomunicacional-en-Ame\%cc\%81ricaLatina-2000-2015.pdf

Mcluhan, M. (1964). Comprender los medios de comunicación. Editorial Lestrobe.

Melero Aguilar, N. (2011). El paradigma crítico y los aportes de la investigación acción participativa en la transformación de la realidad social: un análisis desde las ciencias sociales. Cuestiones Pedagógicas, 21, 339-355.

Observatorio de la democracia (2017, julio 31). ¿Cómo perciben los colombianos a los medios de comunicación? https://obsdemocracia.org/ publicaciones/noticias/como-perciben-loscolombianos-los-medios-de-comuni/

Observatorio de medios (s.f.). Importancia delobservatorio de medios. Universidad de Pamplona. http:// wurw.unipamplona.edu.co/unipamplona/portalIG/ home_52/recursos/01general/22022012/pagina.jsp

Publimetro (2019, diciembre 19). Denuncian censura en entrevista de Iván Duque a Radio Nacional. 
Publimetro. https://www publimetro.co/ co/noticias/2018/12/19/denuncian-censuraentrevista-ivan-duque-radio-nacional.html

Ramos, C. A. (2015, enero-junio). Los paradigmas de la investigación científica. Avances en Psicología, 23(1), 9-17. http://www.unife.edu. pe/publicaciones/revistas/psicologia/2015_1/ Carlos_Ramos.pdf

Rosique Cedillo, G., y Barranquero Carretero, A. (2016). Radiografía de los observatorios de medios en España. Una herramienta ciudadana para la reforma mediática. Revista Latina de comunicación Social, (71), 443-469.

Salazar,H. (2010, febrero 10). Indignación porcierre derevista Cambio. BBC Mundo. https://wurubbc.com/ mundo/america_latina/2010/02/100209_2210_ colombia_revista_gz
Sánchez García, K., y Díaz, O. (2014). ¿Quién vigila a los que vigilan? Una mirada a los Observatorios de Medios en México y sus recursos para la incidencia política. En A. Natal y O. Díaz (coords.). Observatorios ciudadanos. Nuevas formas de participación de la sociedad (pp. 201 a 234). Ediciones Gernika.

Universidad del la Sabana (s.f). Observatorio de Medios de Comunicación [página web]. https://wuwu. unisabana.edu.co/observatoriodemedios/

Uribe, J. (2005). La investigación documental y el estado del arte como estrategias de investigación en ciencias sociales. Estrategias de investigación. Ediciones Universidad Piloto de Colombia.

Voltairenet.org (2006). ¿Qué es el Observatorio de medios? (2006). htto://wuwu.voltairenet.org/ article133328.html 